MS33-P02

\section{Applying fast, accurate lattice energies for molecular crystal structure prediction using CrystalExplorer model energies}

\author{
Peter Spackman ${ }^{1}$, Graeme Day ${ }^{1}$ \\ 1. School of Chemistry, University of Southampton, Southampton, \\ United Kingdom \\ email: p.r.spackman@soton.ac.uk
}

The interaction energy between two molecules can be understood as a sum of several discrete terms [1]: generally electrostatic, polarisation (or induction), dispersion and exchange-repulsion $\left(\mathrm{E}_{\mathrm{tot}}=\mathrm{E}_{\mathrm{ele}}+\mathrm{E}_{\mathrm{pol}}+\mathrm{E}_{\mathrm{dis}}+\mathrm{E}_{\mathrm{rep}}\right)$. CrystalExplorer (CE) model energies [2], calibrated for use in intermolecular interactions, have been shown to estimate experimental crystal lattice energies [4] showing a favourable mean absolute deviation of only $6.6 \mathrm{~kJ} / \mathrm{mol}$ from reference values in 110 crystal structures (the X23, G60, K7 and Z20 benchmark sets).

The relative energies of sets of crystal structures composed of the same molecular building blocks are of particular importance in crystal structure prediction (CSP), as lattice energy ranking is used as a primary predictor for the likelihood of a predicted structure being realized in the laboratory.

We investigate the potential use of CE lattice energies (and variations thereof) in CSP protocols - both in terms of relative energy ordering for different polymorphs and in terms of absolute energies - with reference to density functional and force-field based methods. Using CE lattice energies on the landscape of predicted crystal structures may constitute an accurate technique to improve predicted results, without the associated computational cost (and scaling) of periodic density functional calculations.

Keywords: Lattice Energies, CrystalExplorer, Polymorphism
MS34- Exploring structural dynamics in crystals

Chairs: Dr. Pance Naumov, Prof. Leonard Barbour

MS34-P01

\section{Synthesis, characterization and photochemical properties of a series of new $\mathrm{Ni}$ and $\mathrm{Cu}$ nitro complexes chelated by the $(\mathbf{N}, \mathbf{N}, \mathbf{O})$-type ligands}

Sylwia Kutyła ${ }^{1}$, Radosław Kamiński ${ }^{1}$, Adam Krówczyński ${ }^{1}$, Dominik Schaniel $^{2}$, Katarzyna N. Jarzembskaa ${ }^{1}$

1. Department of Chemistry, University of Warsaw, Żwirki i Wigury 101, 02-089 Warsaw, Poland, Warsaw, Poland

2. CRM2, UMR 7036, Universite' de Lorraine, 54506 Vandoeuvreles-Nancy, France, Vandoeuvre-les-Nancy, France

email: sekutyla@gmail.com

Stimuli-responsive chemical systems in the solid state exhibiting specific photoactive properties have gained a lot of attention nowadays due to their potential technological applications (solar cells, LEDs, data storage assemblies, biological markers, etc.). It is, thus, of great importance to understand the phenomena behind the properties of interest, so as to design the desired materials and sensibly control their properties.

Hence here we present a detailed study of the photo-induced solid state linkage isomerism in a series of new $\mathrm{Ni} / \mathrm{Cu}$ nitro complexes using spectroscopic methods (i.e., UV-vis, IR) physicochemical analyses (e.g., DSC), and advanced crystallographic approaches. The choice of these metals and substrates was dictated by their low price and abundance, which is in contrast to expensive literature-reported $\mathrm{Rh}$ or Pd complexes. [1,2] The desired photoswitchable materials should also be characterised by full conversions (100\%), controllable reversibility, stability and preferably switching observable at temperatures closest to room temperature.

Our Ni complexes exhibit full conversion from the nitro to nitrito form when irradiated with the $590 \mathrm{~nm}$ or $660 \mathrm{~nm}$ LED light at $160 \mathrm{~K}$, whereas the metastable state can be stable up to $240 \mathrm{~K}$. To date, solely the Pd complex reported by Hatcher et al. [3] exhibited similar properties. In turn, the copper systems work best at $10 \mathrm{~K}$, whereas the metastable form is usually stable up to $60 \mathrm{~K}$, which makes them more difficult to be analysed and less applicable materials. It should be emphasized that the newly designed complexes can be relatively easily obtained and modified, and are fully air-stable.

SEK thanks the PRELUDIUM grant (2017/25/N/ST4/02440 of the National Science Centre in Poland for financial support. The authors thank the Wrocław Centre for Networking and Supercomputing (grant No. 285) for providing computational facilities

Figure 1.a) Molecular structure of the $\mathrm{Ni}^{\mathrm{II}}-\mathrm{NO}_{2}$ complex based on the $(\mathrm{N}, \mathrm{N}, \mathrm{O})$ chelating ligand at $100 \mathrm{~K}$, b) Comparison of IR spectra for the ground and metastable state population at $10 \mathrm{~K}$, for $590 \mathrm{~nm}$ LED. 

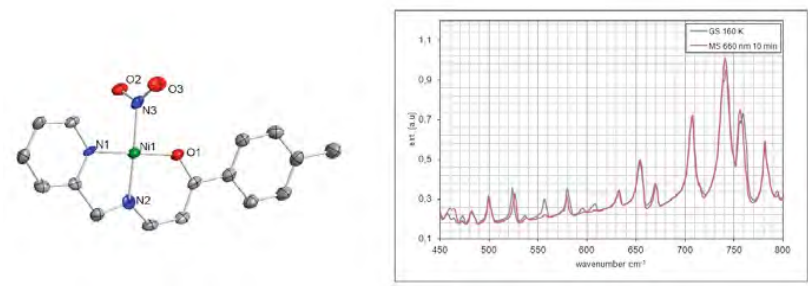

References:

1] Hatcher, L. E.; Christensen, J.; Hamilton, M. L.; Trincao, J.; Allan, D. R.; Warren, M. R.; Clarke, I. P.; Towrie, M.; S. Fuertes; C. C. Wilson; C. H. Woodall; Raithby, P. R., Chem. Eur. J. 2014, 20, $3128-3134$

2] Kovalevsky, A. Y.; King, G.; Bagley, K. A.; Coppens, P., Chem. Eur. J. 2005,11, $7254-7264$.

3] Hatcher, L. E., Cryst. Eng. Comm. 2016, 18, 4180.

Keywords: nitro-complexes, photoswitches, photoisomers

\section{VS34-P02}

\section{An isosymmetric supercritical-like transition in an organic semiconductor}

Arie van der Lee' ${ }^{1}$, Gilles Roche², Guillaume Wantz ${ }^{3}$, Joël Moreau², Olivier Dautel $^{2}$, Jean-Sébastien Filhol ${ }^{2}$

1. Université de Montpellier, Montpellier, France

2. Institut Charles Gerhardt de Montpellier, Laboratoire AM2N, UMR-5253, Université de Montpellier, ENSCM, CNRS, Montpellier, France

3. Université de Bordeaux, IMS, CNRS, Bordeaux, France email: arie.van-der-lee@umontpellier.fr

We show that a high mobility thiophene-based organic semiconductor, BHH-BTBT, has an exceptionally large negative non-linear thermal expansion $\left(-216<\alpha_{2}<-333\right.$ $\mathrm{MK}^{-1}$ ) between 95 and $295 \mathrm{~K}$ along the crystallographic $b$-axis, being compensated by an even larger positive expansion in the perpendicular direction $\left(287<\alpha_{1}<634 \mathrm{MK}^{-1}\right)$ [1]. The temperature evolution of the angle between neighbouring thiophene moieties and the optical band gap show a clear transition around $210 \mathrm{~K}$ but no space group change is detected, i.e. the transition is isosymmetric. A complete theoretical characterization of BHH-BTBT using ab initio molecular dynamics shows that below $\sim 200 \mathrm{~K}$ two different domains exist of which one is dominant but which dynamically coexist around and above $210 \mathrm{~K}$. This transition is reminiscent to the 'diffuse' or 'crossover' phase transition analyzed theoretically by Christy [2] that leads to solid-state supercritical behaviour. The origin of the extreme negative and positive thermal expansion is related to a steric hindrance between adjacent tilted thiophene units and strongly enhanced by attractive $\mathrm{S} \cdots \mathrm{S}$ and $\mathrm{S} \cdots \mathrm{C}$ interactions within the highly anharmonic dynamic mixed-domain phase.

References:

[1] Van der Lee, A, et al, Chem. Sci., accepted, DOI: 10.1039/ C8SC00159F

[2] Christy, A. G. (1995), Acta Cryst. B51, 753-757.

Keywords: thermal expansion, phase transition, supercritical transition 